\title{
Apparent warmth as a function of thermal irradiation'
}

\author{
JOSEPH C. STEVENS AND LAWRENCE E. MARKS \\ JOHN B. PIERCE FOUNDATION LABORATORY AND YALE UNIVERSITY
}

The skin of the back was periodically exposed to a source of radiant heat. In Experiment 1, 20 Ss matched numbers to the apparent warmth aroused by various levels of irradiant flux (method of magnitude estimation). In Experiment 2, 15 Ss matched the loudness of a white noise to the apparent warmth aroused by the same levels used in Experiment 1 (method of cross-modality matching). Both experiments showed that apparent warmth is related to absorbed irradiance by a power function whose exponent is approximately 0.7 .

When the skin is heated by a radiating body such as the sun, a hot fireplace, or a heat lamp, the degree of warmth that is experienced depends on both the intensity and the duration of exposure to the irradiating flux. The present study examines how the degree of experienced warmth depends on the intensity alone, with the duration of each exposure kept short and constant. How the sensation varies with duration is probably a more complex problem; not only does the buildup of warmth proceed slowly compared with most other types of sensory experience, but also the exact course of the buildup appears to differ from one level of the irradiation to another. Ultimately, of course, it would be desirable to understand how warmth varies with both intensity and duration.

The primary question here is whether the warmth aroused by irradiating a large portion of the body (most of the back) obeys the psychophysical power law that describes the functional relation between sensory magnitude $\psi$ and stimulus magnitude $\phi$ on many other sensory continua (S. S. Stevens, 1961). This law says that

$$
\psi=k\left(\phi-\phi_{0}\right)^{\beta}
$$

where $\phi_{0}$ approximates the absolute threshold. The subtraction of $\phi_{0}$ from $\phi$ sets zero on the stimulus scale to coincide with zero on the sensory scale and thereby converts the stimulus into a scale of "distance" above the absolute threshold. It is helpful to express Equation (1) in its logarithmic. form

$$
\log \psi=\log k+\beta \log \left(\phi-\phi_{0}\right)
$$

because in $\log -\log$ coordinates a power function becomes a straight line having a slope equal to $\beta$.

\section{Apparafus and the Nature of the Stimulus}

In Fig. 1, a subject (S) is shown in a chair having in its back a rectangular aperture, $39 \mathrm{~cm}$ wide $\mathrm{x}$ $32 \mathrm{~cm}$ high. From time to time, the bare surface of the S's back exposed to this aperture was Irradiated by four heat lamps located $3 \mathrm{ft}$ behind the S. The exposure duration was controlled at $5 \mathrm{sec}$ by means of the hand-operated metal shutter.

The four lamps were arranged to cast homogeneous flux on the skin. Each lamp was a General Electric T-3 quartz lamp, $40 \mathrm{~cm}$ long, mounted in a parabolic-cylindrical reflector made of stainless steel.

The radiant intensity was controlled by varying the voltage across the lamps. The relation between the voltage applied and the level of flux emitted was determined with a calibrated Hardy radiometer placed at the aperture of the chair. Of course, only part of the flux that irradiates the skin is actually absorbed; moreover, the proportion of the flux absorbed varies with the color temperature of the lamps and consequently with the voltage applied. As the voltage is increased, the peak of the emitted spectrum shifts to shorter wavelengths, which are less readily absorbed by the skin.

Fortunately, the reflectance curve for human skin has been measured (Hardy, Hammel, \& Murgatroyd, 1956), and its complement (the absorption curve) can be used to weight the spectrum emitted by a source in order to determine, for each wavelength, the irradiance absorbed by the skin. The integral of the weighted spectrum gives the total absorbed

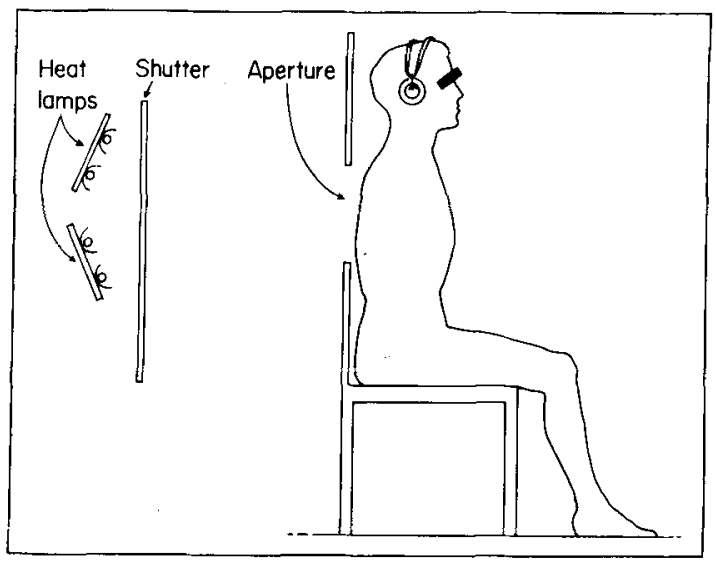

Fig. 1. The experimental setup. 


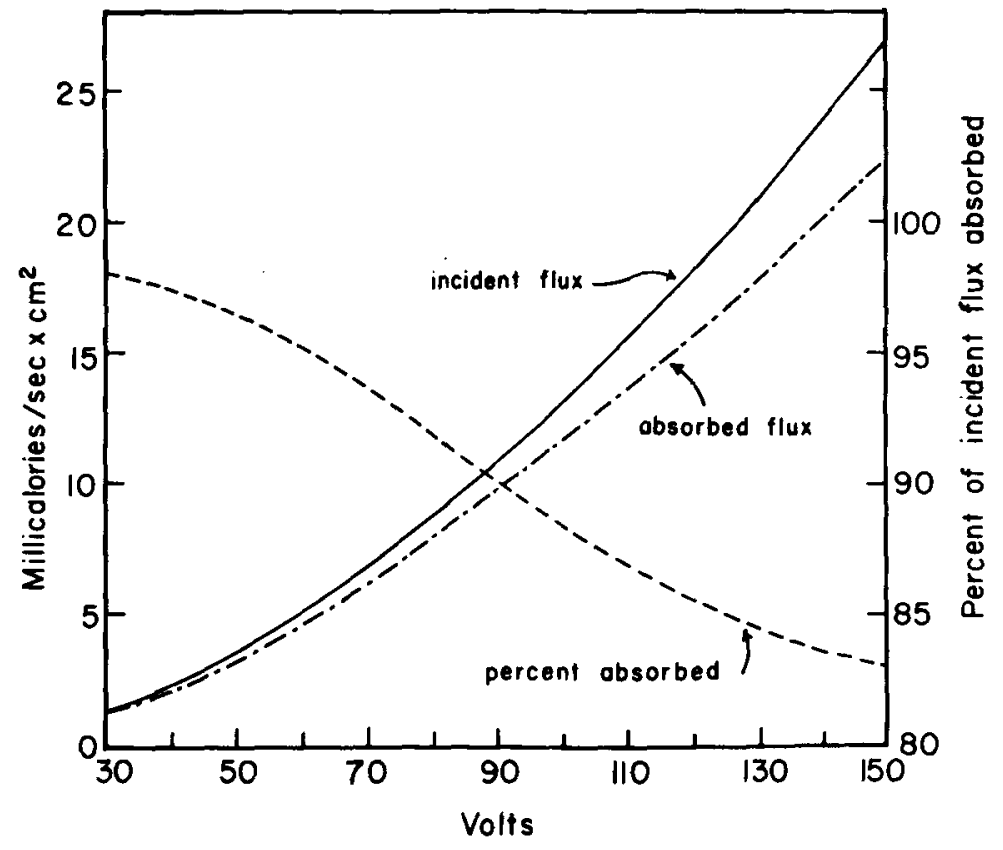

Fig. 2. The left-hand ordinate gives the incident and the absorbed flux as a function of voltage applied to the $T-3$ quartz lamp. The right-hand ordinate gives the percentage of the total incident flux absorbed by the skin. flux. This quantity has been computed by Gagge, Rapp, and Hardy (1967) for many levels of voltage applied to the T-3 quartz lamp. In Fig. 2 (righthand ordinate) the ratio of the absorbed irradiance to the total irradiance is shown as a function of voltage. At $30 \mathrm{~V}$, for example, nearly all (98\%) of the incident flux is absorbed, but at $150 \mathrm{~V}$ only $83 \%$ is absorbed. Figure 2 also shows how the total irradiance and the total absorbed irradiance (in mcal/ sec $x \mathrm{~cm}^{2}$ ) each depend upon the voltage. Table 1 gives the levels of voltage, irradiance, and absorbed irradiance in the present experiments. These levels produced sensations that range from just noticeably warm to very warm, but not painful.

One complication in the measurement of the stimulus derives from the relation between wavelength and penetration into the skin. Although essentially

Table 1. The voltages across the lamps ( $V$ ), the associated incident fux (H) and the associated absorbed flux in mcal $/ \mathrm{sec} \times \mathrm{cm}^{2}$ $(\phi)$; the mean $(\overline{\mathrm{x}})$, standard deviation $(\sigma)$, and the adjusted standard deviation $\left(\sigma^{\prime}\right)$ of the magnitude estimations; the mean $(\overline{\mathrm{dB}})$, the standard deviation of $\left(\sigma_{\mathrm{dB}}\right)$ and the adjusted standard deviation $\left(\sigma^{\prime} \mathrm{dB}\right.$ ) of the settings (in $\mathrm{dB}$ re 0.0002 microbar) in cross-nodality matching.

\begin{tabular}{rcccccccc}
\multicolumn{3}{c}{$\begin{array}{c}\text { Stimulus } \\
V\end{array}$} & $\mathbf{H}$ & $\boldsymbol{\phi}$ & \multicolumn{3}{c}{ Magnitude estimation } & \multicolumn{3}{c}{ Loudness matching } \\
\hline 50 & 3.50 & 3.38 & 5.50 & 0.38 & 0.20 & 38.8 & 14.2 & 5.9 \\
60 & 4.90 & 4.66 & 7.43 & 0.36 & 0.17 & 42.6 & 14.1 & 4.5 \\
70 & 6.90 & 6.45 & 10.3 & 0.30 & 0.14 & 47.9 & 12.6 & 4.1 \\
90 & 11.0 & 9.90 & 13.8 & 0.27 & 0.11 & 50.2 & 14.8 & 4.5 \\
110 & 15.5 & 13.5 & 17.6 & 0.31 & 0.11 & 53.2 & 14.9 & 4.2 \\
130 & 21.1 & 17.8 & 21.7 & 0.29 & 0.11 & 57.9 & 14.2 & 3.9 \\
150 & 27.1 & 22.5 & 26.6 & 0.29 & 0.15 & 61.7 & 14.9 & 4.9 \\
\hline
\end{tabular}

all of the infrared greater than $3 \mu$ is absorbed within $0.2 \mathrm{~mm}$ of the skin surface, $40 \%$ of the absorbed infrared $(0.75-3 \mu)$ and $50 \%$ of visible light $(0.35-0.75 \mu)$ penetrate beyond $0.2 \mathrm{~mm}$; both visible light and penetrating infrared are somewhat less effective than nonpenetrating infrared in the arousal of just-detectable warmth (Oppel \& Hardy, 1937). Since the peak of the spectrum of the stimulus used in the present experiment varied with level, the relative proportion of penetrating to nonpenetrating radiation also varied. It is probable, however, that this variation was minor or negligible, since Stolwijk and Hardy (1965) found the rise in temperature of the skin surface to be proportional to absorbed irradiance produced by the lamp used in the present study.

\section{Procedure}

Two experiments were run. The first used the method of magnitude estimation, the second the method of cross-modality matching. Each exposure to the heat lasted $5 \mathrm{sec}$, and about $30 \mathrm{sec}$ separated successive exposures. (A 30-sec pause between exposures is necessary to maintain the skin in a reasonably uniform state of sensitivity, even though the sensation of warmth appears to dissipate almost instantly following the offset of stimulation.) Just before an exposure the $S$ was alerted. The $S$ was blindfolded in order to prevent visual cues. Ambient temperature was held constant at $70^{\circ} \mathrm{F}$.

(1) Magnitude Estimation. First, two exposures (at intermediate levels) were given to acquaint the $\mathrm{S}$ with the stimulus. These exposures were not judged. Then one of the levels corresponding to 70 , 
90, or $110 \mathrm{~V}$ (see Table 1) was presented, and the $S$ assigned any number that seemed to him convenient to stand for the degree of warmth experienced. This judgment was not counted. To subsequent exposures the $S$ attempted to assign numbers proportional to the degree of warmth experienced. All the Ss were familiar with the method of magnitude estimation and had previously followed this procedure in at least one experiment on another sense modality.

The order of the stimulus levels was irregular and different for each $S$, and each level was presented twice in the course of the experimental session.

Twenty white adults, including the authors, served as Ss.

(2) Cross-Modality Matching. Instead of assessing the warmth numerically, the $S$ adjusted the loudness of a noise to "match" the degree of apparent warmth. Otherwise the procedure was substantially the same as in the first experiment.

The signal from a white noise generator was filtered $(75-4800 \mathrm{~Hz})$, passed through a potentiometer (adjusted by the $S$ to effect the match), and fed into a pair of calibrated Grason-Stadler P1D18 earphones mounted in $\mathrm{MX}-41 / \mathrm{AR}$ cushions. The voltage across the earphones was recorded graphically throughout the session. The potentiometer (called a "sone potentiometer') was designed to give approximately equal increments of apparent loudness for equal increments of dial rotation. The circuit and calibration are described by J. C. Stevens and Guirao (1964).

The Ss were instructed to set the noise alternately to levels too loud and too soft to be correct, and to "zero in" on the final setting. It usually took only a few seconds for the $S$ to effect the match. Fifteen persons took part. Each had previously served in the first experiment.

\section{Results}

Magnitude Estimation. In Table 1 are the geometric means of the estimates and the standard deviations of the logarithms of the estimates. The variability of the logarithmically transformed estimates is practically constant across stimulus level (except for slightly higher values at the low end of the range); this implies that the variability of the estimates (not transformed) is approximately proportional to the apparent magnitude.

A large proportion of the variability is attributable to the fact that each observer estimated in terms of his own "modulus," i.e., chose any number he deemed convenient to stand for the apparent warmth aroused by the first exposure. This component of the variability, sometimes called the "intercept" component, can be partialled out (Lane, Catania, \&
S. S. Stevens, 1961). The method involves the following computations on the logarithms of the estimates: (1) the grand mean of all the estimates across stimuli; (2) the mean of each S's estimates across stimuli; and (3) the difference between the grand mean and each S's mean. For each $S$ this difference (3) is then added to each of his estimates. Finally, the geometric mean and the standard deviation across $\mathrm{Ss}$ is computed for each stimulus level. The geometric means of the adjusted estimates are identical to those of the unadjusted estimates, but the standard deviations (see Table 1) are, on the average, Iess than half as large as the original standard deviations. Of course, the variability could have been reduced in the first place by instructing each $S$ to use the same modulus, but long experience with the method of magnitude estimation suggests that it is better not to impose constraints of this type on the observer.

Even the adjusted variability is fairly large, and most Ss thought the task was difficult. To the authors, the sensations of warmth seemed to be rather subtle and difficult to assess quantitatively.

Nevertheless, the average results are orderly and are consistent with the psychophysical power law. The geometric means are plotted in the log-log coordinates of Fig. 3 (as a function of the calculated absorbed flux $\phi$ ) and of Fig. 4 (as a function of the difference between the absorbed flux of the exposure $\phi$ and the absorbed flux that approximates the absolute threshold for warmth $\phi_{0}$-about $1.0 \mathrm{mcal} /$ $\sec \times \mathrm{cm}^{2}$ ). In Fig. 3 the function is slightly curvilinear (concave downward), a shape to be expected if $\phi_{0}$ is not subtracted from each stimulus level. In Fig. 4, however, the function becomes rectilinear. The equation of the line is $\log \psi=0.48+0.7 \log (\phi-1.0)$, or in its nonlogarithmic form $\psi=3.0(\phi-1.0)^{0.7}$.

The constants of the function in Fig. 4 were determined by the following analytic procedure. For each of several hypothetical values of $\phi_{0}$ a straight line was fitted by the method of least squares, operating on the logarithms of the geometric means and the logarithms of $\phi-\phi_{0}$. Then, for each line thus determined, the Pearson coefficient of correlation was computed to show the strength of the relation between the logs of the geometric means and the logs of $\phi_{-} \phi_{0}$. Figure 5 shows how the constants $\log k$ and $\beta$ of the fitted lines and the Pearson coefficient $r$ depend upon the assumed values of $\phi_{0}$. The highest correlation (0.9994) was obtained when $\phi_{0}$ equals 1.0. At this value the slope constant $\beta$ equals 0.7 and the intercept constant $\log \mathrm{k}$ equals 0.48 .

Thus the absolute threshold was not measured by means of the conventional psychophysical procedures. The value of $1.0 \mathrm{mcal} / \mathrm{sec} \times \mathrm{cm}^{2}$ is commensurate with some causal threshold measurements 


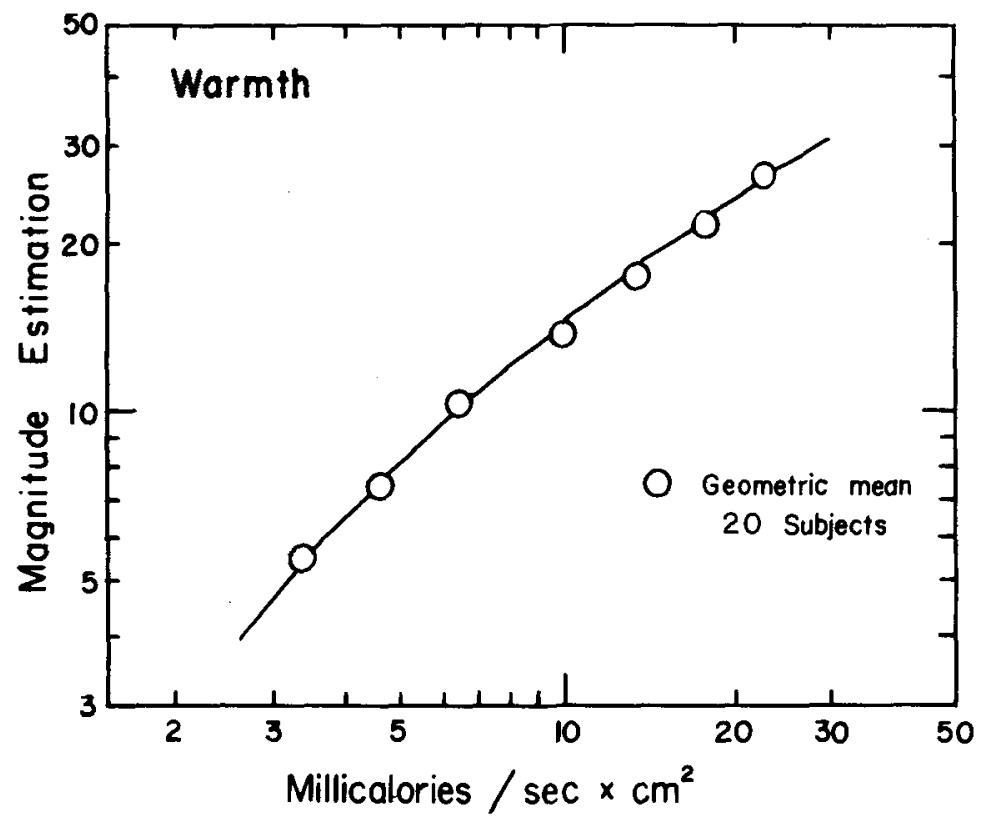

Fig. 3. Magnitude estimation of warmth as a function of the calculated irradiant flux absorbed by the skin.

made on the authors. It is higher than a value (about

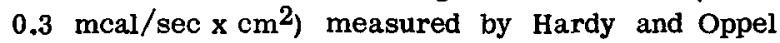
(1937) who used a very "sensitive" psychophysical procedure, and lower than a value (about $1.6 \mathrm{mcal} /$ sec $\times \mathrm{cm}^{2}$ ) measured by Chrenko (1964) for a comparable area of the skin. Of course, the "effective" threshold undoubtedly varied to some extent from one observer to another, and even on the average would presumably differ from the threshold measured by a different psychophysical procedure. Nevertheless, experiments on a variety of sensory continua have shown that the value of $\phi_{0}$ necessary to rectify the psychophysical function in $\log -\log$ coordinates is roughly equivalent to the more conventional measurements of the absolute threshold (J. C. Stevens \& S. S. Stevens, 1962).
The value of $k$ has little interest here since it depends on the arbitrary selection of the units of measurement. The exponent $\beta$ is not dependent on the units chosen. Its size (0.7) implies that the sensation of warmth in the back grows as a decelerating function of the stimulus level.

Cross-Modality Matching. For each level of the stimuius, Table 1 gives the mean setting (in $\mathrm{dB}$ re 0.0002 microbar), the standard deviation, and the standard deviation after the intercept variability has been partialled out.

If two sensory continua $a$ and $b$ are described by the two power functions,

$$
\psi_{\mathrm{a}}=\mathrm{k}_{\mathrm{a}} \phi_{\mathrm{a}}^{\alpha} \text { and } \psi_{\mathrm{b}}=\mathrm{k}_{\mathrm{b}} \phi_{\mathrm{b}}{ }^{\beta} \text {, }
$$

(where $\phi$ is measured as distance above the absolute
Fig. 4. Magnitude estimation of warmth as a function of the absorbed flux corrected by subtracting the threshold nux $\left(1.0 \mathrm{mcal} / \mathrm{sec} \times \mathrm{cm}^{2}\right)$ from each stimulus level. The vertical lines are interquartile ranges calculated after the intercept variability had been removed.

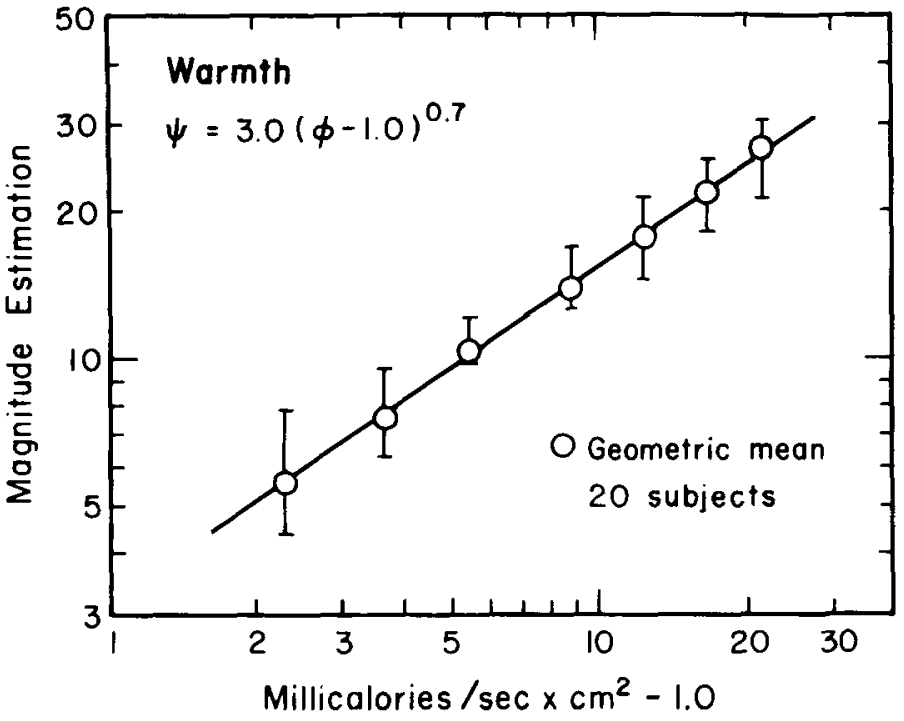

Perception \& Psychophysics, 1967, Vol. 2 (12) 


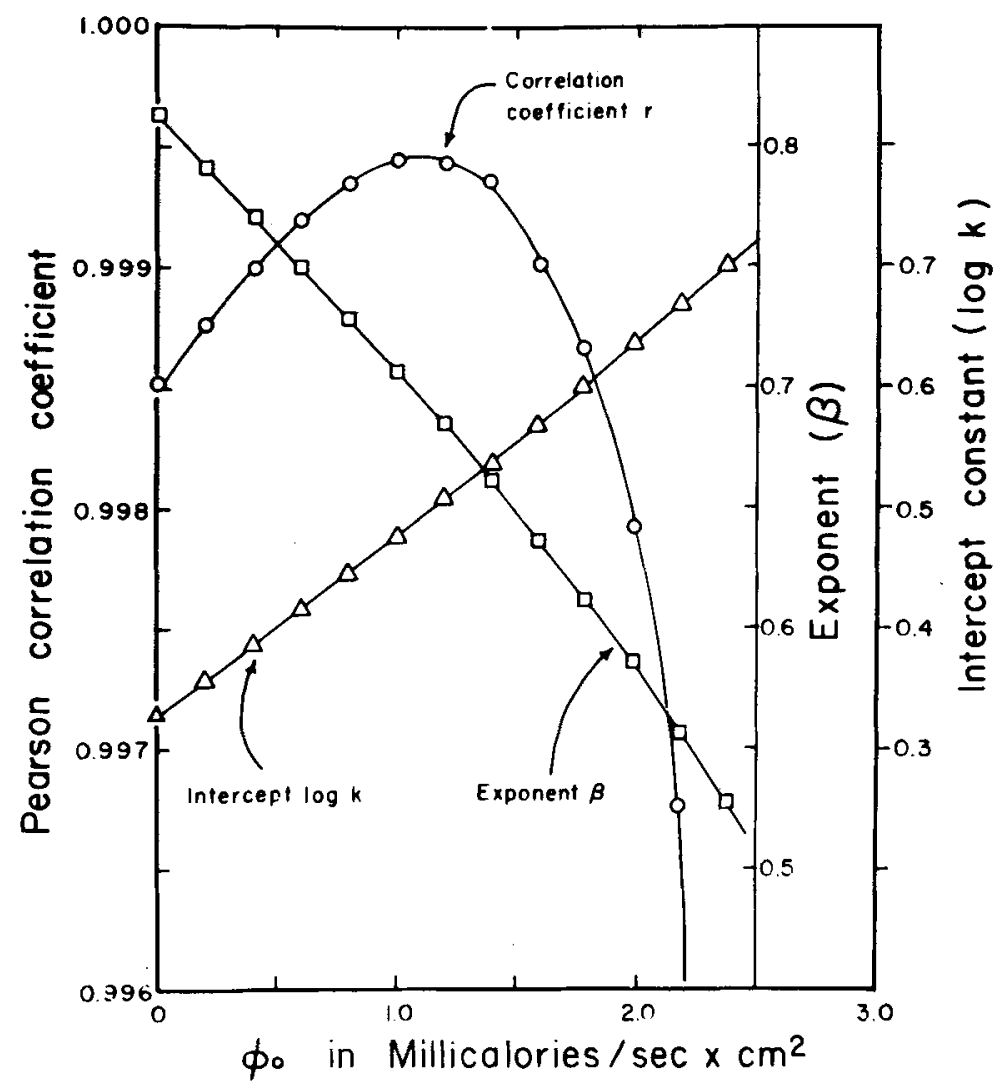

Fig. 5. How the correlation coefficient $r$, the exponent $\beta$, and the proportionality constant $\log k$ depend on the assumed value of the threshold constant $\phi_{0}$ for magnitude estimation of warmth. threshold), and if the task is to match $\psi_{a}$ to $\psi_{b}$ at various levels (as in the present experiment when loudness is matched to apparent warmth), the resulting equal-sensation function ought to have the equation

$$
\phi_{\mathrm{a}}=\frac{\mathbf{k}_{\mathbf{b}}}{\mathbf{k}_{\mathrm{a}}} \phi_{\mathbf{b}} \beta / \alpha
$$

Thus the prediction is that the equal-sensation function will be a power function whose exponent equals $\beta / \alpha$. The exponent $\alpha$ of the psychophysical power function relating loudness to sound pressure is 0.6 (S. S. Stevens, 1966), so the exponent $\beta / \alpha$ predicted in the present experiment is $0.7 / 0.6$ or 1.17 .

As a test of this prediction, the mean decibel settings of the noise have been plotted in Fig. 6 as a function of the same stimulus scale used in Fig. 4 $-\left(\mathrm{mcal} / \mathrm{sec} \times \mathrm{cm}^{2}\right)-1.0$. Both coordinates are logarithmic (the decibel scale is a logarithmic scale). Since the level of the loudness threshold is negligible compared to the average sound pressure levels set by the $S$, it was unnecessary to correct the ordinate of Fig. 4 by the subtraction of a threshold constant. The average settings lie fairly close to the straight line, which has been drawn to have the predicted slope, 1.17. This result helps to substantiate (1) the power law for apparent warmth, (2) the approximate value of the exponent $(0.7)$ measured by magnitude estimation, and (3) the approximate value of $\phi_{0}(\mathbf{1 . 0})$. The present application of the method of cross-modality matching is one of dozens that have succeeded in verifying the psychophysical power law (see, for example, J. C. Stevens, Mack, \& S. S. Stevens, 1960, and S. S. Stevens, 1966).

\section{Relation to Previous Studies}

A pioneer attempt to measure the psychophysical relation between apparent warmth and irradiance was undertaken by Herget, Granath, and Hardy (1941) more than a decade before S. S. Stevens proposed the psychophysical power law. In their experiment, a spot on the blackened forehead was irradiated for 2 sec at a time, and the .S attempted to rate the resulting sensation on a scale from zero to 10 . The $S$ was told to assign the number 10 to the apparent warmth of the first stimulus, the most intense of the series. Figure 7 is a $\log -\log$ plot of the average estimations of the two Ss tested. A power function was fitted to the data by the iterative least-squares method described above. The highest correlation coefficient $(0.992)$ was obtained when $\phi_{0}$ equals 1.7 and $\beta$ equals 0.54 . This result agrees fairly well with the present study, given the procedural differences between the two experiments.

A quite different result was obtained when small 


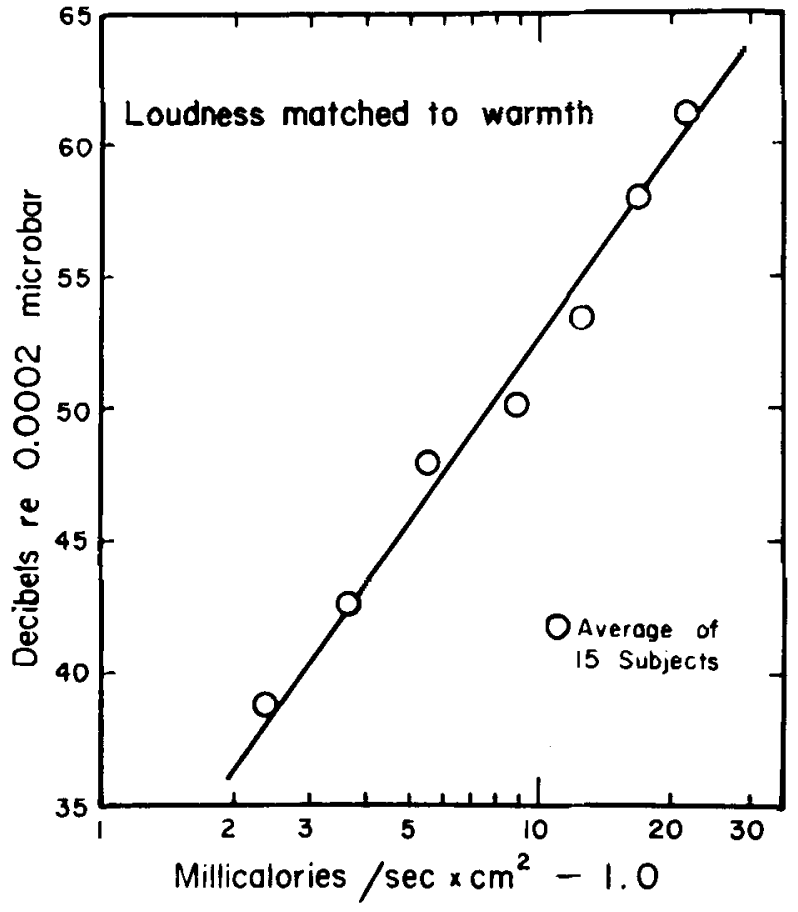

Fig. 6. Equal-sensation function obtained by cross-modality matching of loudness to warnth. The abscissa is the absorbed flux corrected by subtracting the calculated threshold flux $(1.0 \mathrm{mcal} / \mathrm{sec}$ $x \mathrm{~cm}^{2}$ ). The line through the data has been drawn with the slope predicted for these cross-modality matches.

metal stimulators at different temperatures were touched briefly to the forearm (J. C. Stevens \& S. S. Stevens, 1960; J. C. Stevens, Mack, \& S. S. Stevens, 1960). The S's task was either (1) to assign numbers proportional to the degree of thermal sensation aroused (magnitude estimation), or (2) to exert on a hand dynamometer a force that seemed to match the thermal sensation (cross-modality matching). The exponent of the psychophysical power function turned out to be about 1.6 for warmth and about 1.0 for cold.

Why heat stimulation by conduction from the metal stimulators should yield an exponent at least twice as large as that obtained with irradiant stimulation is one of many questions in the area of thermal sensitivity that call for investigation. Other questions include the effect on the psychophysical function of such parameters as duration, and bodily locus of stimulation. So far, the evidence suggests that thermal sensations obey the psychophysical power law under a variety of conditions, but much remains to be learned about the range of conditions under which the law holds and about how the constants of the power function depend on the parameters of stimulation.

\section{References}

Chrenko, F. A. Threshold intensities of thermal radiation evoking sensations of warmth. J. Physiol., 1964, 173, 1-12.

Gagge, A. P., Rapp, G. M., \& Hardy, J. D. Effective radiant field and operative temperatures necessary for comfort in the radiant heating. Transactions of the American Society of Heating, Refrigeration, and Airconditioning Engineers. 1967, 73, Part I.

Hardy, J. D., Hammel, H. T., \& Murgatroyd, D. Spectral transmittance and reflectance of excised human skin. J. appl, Physiol., $1956,9,257-264$.

Hardy, J. D., \& Oppel, T. W. Studies in temperature sensation. III. The sensitivity of the body to heat and the spatial summation of the end organ responses. J. clin. Invest., 183?, 16, 533-540.

Herget, C, M., Granath, L. P., \& Hardy, J. D. Thermal sensation and discrimination in relation to intensity of stimulus. Amer. J. Physiol., 1941, 134, 645-655.

Lane, H. L., Catania, A. C., \& Stevens, S. S. Voice level: Autophonic scale, perceived loudness, and effect of sidetone. $J$. Acoust. Soc. Amer., 1961, 33, 160-167.

Oppel, T. W., \& Hardy, J. D. Studies in temperature sensation. I. A comparison of the sensation produced by infra-red and visible radiation. J. clin. Invest., 1937, 16, 517-524.

Stevens, J. C., \& Guirao, M. Individual loudness functions. $I$. Acoust. Soc. Amer., 1964, 36, 2210-2213.

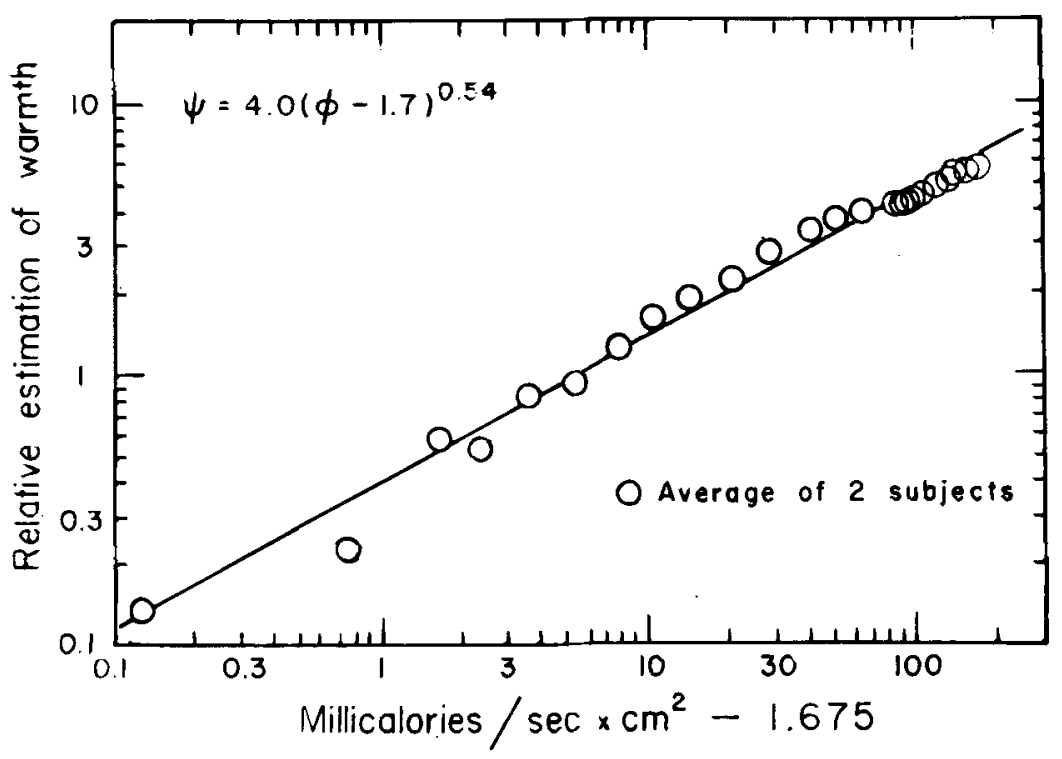

Fig. 7. The average estimations of warmth by the two subjects tested by Herget, Granath, and Hardy, 1941 (read trom their graph, p 648). This plot combines the results from two different runs, one on the upper portion of the stimulus range, the other on the lower. 
Stevens, J. C., Mack, J. D., \& Stevens, S. S. Growth of sensation on seven continua as measured by force of handgrip. J. exp. Psychol., 1960, 59, 60-67.

Stevens, J. C., \& Stevens, S. S. Warmth and cold: Dynamics of sensory intensity. J. exp. Psychol., 1960, 60, 183-192.

Stevens, J. C., \& Stevens, S. S. Physiological zero and the psychophysical law. In Proc. of the 16th int. Cong. of Psychol. Amsterdam: North Holland, 1962, 192-193.

Stevens, S. S. To honor Fechner and repeal his law. Science, $1961,133,80-86$

Stevens, S. S. Matching functions between loudness and ten other continua. Percept. \& Psychophys., 1966, 1, 5-8.

Stolwijk, J. A. J., \& Hardy, J. D. Skin and subcutaneous temperature changes during exposure to intense thermal radiation. $J$. appl. Physiol., 1965, 20, 1006-1013.

\section{Note}

1. This research was supported by Contract F44620-67-C-0017 with the Air Force Office of Scientific Research. Reproduction for any purpose of the United States Government is permitted.

(Accepted for publication August 15, 1967.) 